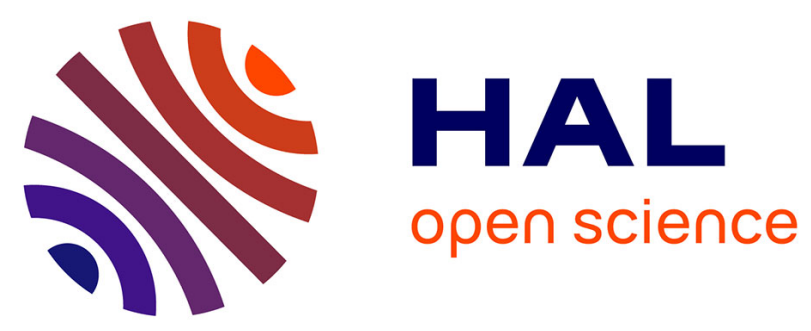

\title{
Dilatational and Compacting Behavior around a Cylindrical Cavern Leached Out in a Solid-Fluid Elastic Rock Salt
}

\author{
Giulio Sciarra, Francesco Dell'Isola, Kolumban Hutter
}

\section{- To cite this version:}

Giulio Sciarra, Francesco Dell'Isola, Kolumban Hutter. Dilatational and Compacting Behavior around a Cylindrical Cavern Leached Out in a Solid-Fluid Elastic Rock Salt. International Journal of Geomechanics, 2005, pp.11. hal-00502133

\section{HAL Id: hal-00502133 \\ https://hal.science/hal-00502133}

Submitted on 13 Jul 2010

HAL is a multi-disciplinary open access archive for the deposit and dissemination of scientific research documents, whether they are published or not. The documents may come from teaching and research institutions in France or abroad, or from public or private research centers.
L'archive ouverte pluridisciplinaire HAL, est destinée au dépôt et à la diffusion de documents scientifiques de niveau recherche, publiés ou non, émanant des établissements d'enseignement et de recherche français ou étrangers, des laboratoires publics ou privés. 


\title{
Dilatational and Compacting Behavior around a Cylindrical Cavern Leached Out in a Solid-Fluid Elastic Rock Salt
}

\author{
Giulio Sciarra'; Francesco dell'Isola ${ }^{2}$; and Kolumban Hutter ${ }^{3}$
}

\begin{abstract}
A fluid-filled cylindrical cavern of circular cross section in a homogeneous infinite fluid-saturated polycristalline (salt) formation subjected to isotropic stress is set under internal pressure that differs from the confining pressure. The fluid in the cavern and in the mixture is treated as ideal and the solid as elastic. The state of stress that is established as a consequence of an outside pressure and a cavern pressure serves as the reference state. Perturbing the cavern pressure induces small changes in the solid and fluid densities and in the solid displacements. We compute these and other fields as functions of the radial distance from the cavern center and show that, depending on the relative stress levels, the (salt) formation experiences either a dilatation or a compaction that is highly concentrated in a thin boundary layer near the cavern wall and tapers off as one moves away from it. The amount of dilatation/compaction of the cylindrical wall and the thickness of the boundary layer grow with an increase in the difference between the referential confining pressure and the pressure in the cavern.
\end{abstract}

DOI: 10.1061/(ASCE)1532-3641(2005)5:3(233)

CE Database subject headings: Salt domes; Dilatancy; Compaction; Mixtures; Prestressing; Elastic analysis; Pressures.

\section{Introduction}

In geotechnique, caverns excavated in salt domes are used as storage volumes for the disposal of waste and hydrocarbons. These caverns, leached out in salt formations, are characterized by a damaged zone in the vicinity of their surface. In fact, permeability measurements (Cosenza et al. 1999; Stormont 1997) in the immediate vicinity of the cavern walls disclose a dilatation of the pores within a relatively small boundary layer of the polycrystalline salt. The origin of this disturbed rock zone is still somewhat debatable; however, a microstructured theory of solid-fluid mixtures was recently proposed (Sciarra 2002; Sciarra et al. 2001, 2003; dell'Isola et al. 2000) that offered a means of explaining, in the framework of an elastic model, the occurrence of the apparently counterintuitive expansion of the pore-space by the increase in internal cavern pressure. In our initial publication on this subject (Sciarra et al. 2001), second-gradient effects for the deformed porous salt matrix, which was filled with an ideal fluid, were claimed to be essential. Our present understanding tells us that second-gradient effects yield dominant qualitative behavior of the dilatancy deformation of the salt-fluid mixture only for specific

${ }^{1}$ Doctor, Dipartimento di Ingegneria Chimica, dei Materiali, delle Materie Prime e Metallurgia, Univ. of Rome "La Sapienza," via Eudossiana 1800184 Rome, Italy. E-mail: giulio.sciarra@uniroma1.it

${ }^{2}$ Professor, Dipartimento di Ingegneria Strutturale e Geotecnica, Univ. of Rome "La Sapienza," via Eudossiana 1800184 Rome, Italy. E-mail: francesco.dellisola@uniroma1.it

${ }^{3}$ Professor, Dept. of Mechanics, Darmstadt Univ. of Technology, D-64289 Darmstadt, Germany. E-mail: hutter@mechanik.tu-darmstadt.de

Note. Discussion open until February 1, 2006. Separate discussions must be submitted for individual papers. To extend the closing date by one month, a written request must be filed with the ASCE Managing Editor. The manuscript for this paper was submitted for review and possible publication on March 15, 2004; approved on December 9, 2004. This paper is part of the International Journal of Geomechanics, Vol. 5, No. 3, September 1, 2005. CASCE, ISSN 1532-3641/2005/3-233-243/ $\$ 25.00$. simple load configurations, while in general it affects the deformation field only quantitatively, for instance by altering the dimension of dilatancy boundary layers.

To understand this, consider a cylindrical, very long cavern surrounded by an infinite salt-brine mixture; let us model it as a first-gradient elastic material and assume that the cylinder is subject to a steady internal pressure $p_{01}$, while the outside boundary, ideally at infinity, is carrying the isotropic pressure $p_{02}$ (Fig. 1). Because both the cavern pressure and the outer boundary pressure, integrated over their individual closed boundaries, are in static equilibrium, $p_{01}$ and $p_{02}$ can individually be chosen; they thus define a multitude of isotropic stresses states. In particular, fluid can be pressed into or sucked from the cylinder, and the effect of such actions may be studied. Therefore, replacing $p_{01}$ by $p_{01}+\Delta p_{01}$ (and applying the zero displacement condition at infinity) will, for a given $p_{02}$, change the deformation state in the solid-fluid mixture around the fluid-filled cavern, in particular inducing some dilatancy boundary layers inside the solid matrix. This deformation state will depend upon the prestresses $p_{01}$ and $p_{02}$. Of concern is how the apparent solid density $\Delta \rho_{s}$ changes due to the imposed additional $\Delta p_{01}$ as a function of the radial distance from the cavern wall.

For the first-gradient solid-fluid mixture model used in the present paper, the results are displayed in Fig. 2. If $p_{01}<p_{02}$, a linear increase in the cavern pressure results in a dilatancy, $\Delta \rho_{s}<0$, for all $r \in[a, \infty)$, where $a=$ radius of the cylinder; alternatively, when $p_{01}>p_{02}$, an increase in the cavern pressure will result in a compaction, $\Delta \rho_{s}>0$, for all $r \in[a, \infty)$. These results imply almost by default that, for $p_{01}=p_{02}, \Delta \rho_{s}=0$ for all $r \in[a, \infty)$. This is indeed the result that motivated us to improve the first-gradient model by a second-gradient model in our earlier paper (Sciarra et al. 2001) to recover dilatancy effects also in the wall-near boundary layer when $p_{01}=p_{02}$.

The monotonic behavior exhibited by the graphs of Fig. 2 prevails for all prestress conditions $p_{01}$ and $p_{02}$. However, as $p_{01}$ increases beyond $p_{02}$, the maximum value of $\Delta \rho_{s}$ at $r=a$ increases and the boundary layer becomes thicker (Fig. 3). 


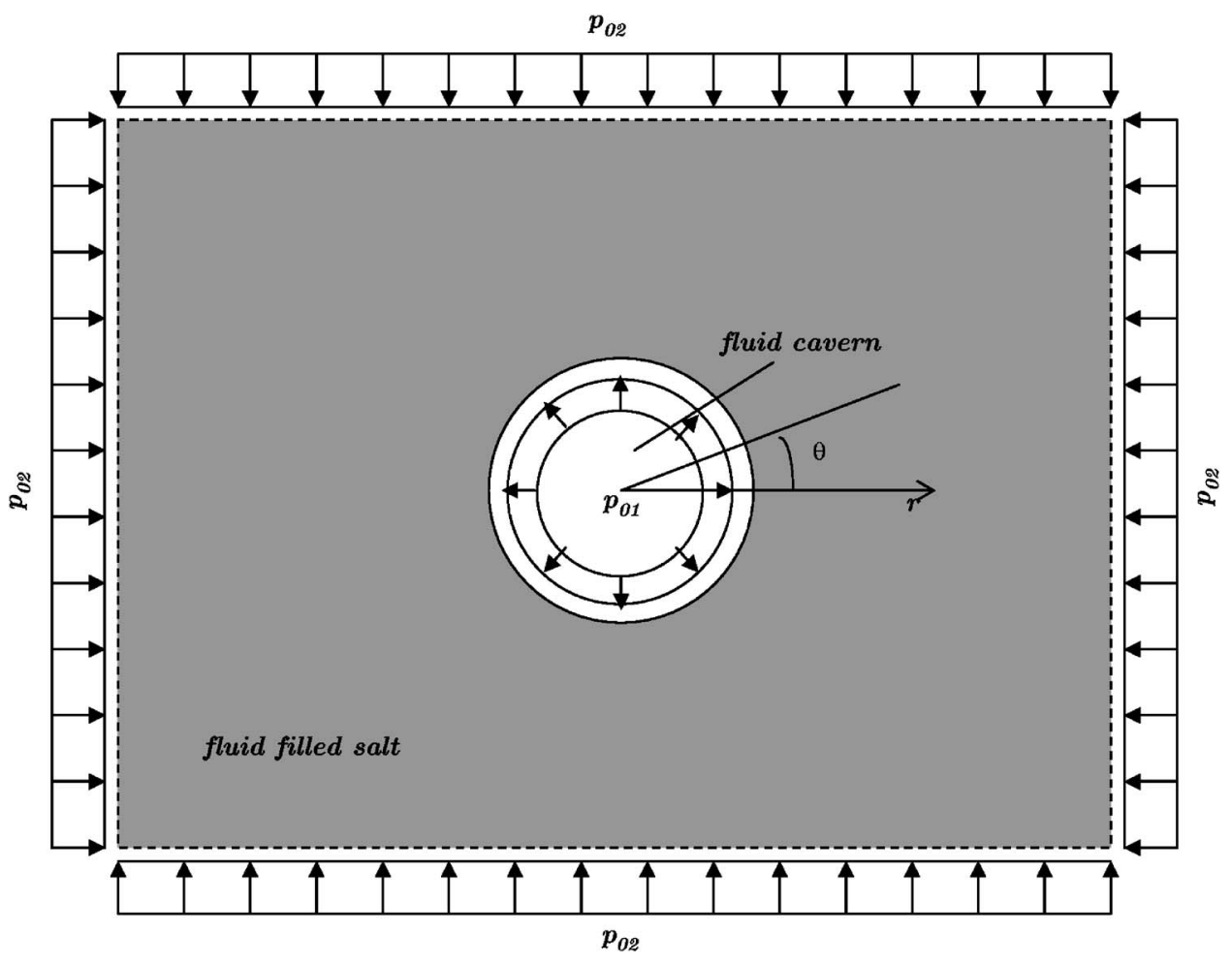

Fig. 1. Cylindrical cavern of radius $a$ filled with an inviscid fluid, in a solid-fluid mixture of polycrystalline salt and brine. The porous salt has a very low permebility. Plane deformation is assumed.

\section{Formulation of Problem}

Consider a solid-fluid mixture; identify the solid and fluid constituents by the labels $s$ and $f$, respectively. Material particles of the fluid and the solid are identified, respectively, by their position vectors $\mathbf{X}_{f}$ and $\mathbf{X}_{s}$ in fixed reference configurations $\Omega_{f}^{0}$ and $\Omega_{s}^{0}$. As is usually done in the theory of mixtures, we presume that, at any time $t$, particles of both constituents occupy the same position $\mathbf{x}$ in the present configuration $\Omega$. The velocity $\mathbf{v}_{a}(a=f, s)$ of the material particle $\mathbf{X}_{a}$ is defined by

$$
\mathbf{v}_{a}=\frac{d_{a} \mathbf{u}_{a}\left(\mathbf{X}_{a}, t\right)}{d t}
$$

where $d_{a} / d t=$ material time derivative following the motion of $\mathbf{X}_{a}$; and $\mathbf{u}_{a}=$ displacement of the $a$ th constituent from its reference configuration. Let $\rho_{f}$ and $\rho_{s}$ denote, respectively, the apparent mass densities of the fluid and the solid; then the mass density, $\rho$, of the mixture equals $\rho_{f}+\rho_{s}$. The mean barycentric velocity $\mathbf{v}$ of the mixture is defined by

$$
\rho \mathbf{v}=\rho_{f} \mathbf{v}_{f}+\rho_{s} \mathbf{v}_{s}
$$

We assume the reader to be familiar with the concepts of the theory of mixtures. Details can be taken from the literature, e.g., Truesdell (1957); Müller (1985); and Hutter and Jöhnk (2003).

\section{Balance Laws}

We assume a classical mixture of first-gradient formulation and use the principle of virtual power to derive the balance of linear momentum and the boundary conditions for each constituent; that is

$$
\begin{aligned}
\int_{\Omega} & \left(\mathbf{m}_{s} \cdot \mathbf{v}_{s}+\mathbf{m}_{f} \cdot \mathbf{v}_{f}+\mathbf{T}_{s} \cdot \nabla \mathbf{v}_{s}-p_{f} \operatorname{div} \mathbf{v}_{f}\right) d V \\
\quad= & \int_{\Omega}\left(\mathbf{b}_{s} \cdot \mathbf{v}_{s}+\mathbf{b}_{f} \cdot \mathbf{v}_{f}\right) d V+\int_{\partial \Omega}\left(\mathbf{t}_{s} \cdot \mathbf{v}_{s}+\mathbf{t}_{f} \cdot \mathbf{v}_{f}\right) d A
\end{aligned}
$$

Here, $\mathbf{m}_{a}=$ bulk solid-fluid interaction force; $\mathbf{T}_{s}=$ partial Cauchy stress in the solid; $p_{f}=$ hydrostatic pressure in the fluid (we assume that the partial Cauchy stress in the fluid is spherical); $\nabla=$ gradient operator with respect to coordinates in the present configuration; $\mathbf{b}_{a}=$ density of partial body forces; and $\mathbf{t}_{a}=$ partial surface tractions. The objectivity of the left-hand side of Eq. (3) implies that the sum of the two internal supplies $\mathbf{m}_{s}$ and $\mathbf{m}_{f}$ of linear momentum equals 0 and $\mathbf{T}_{s}$ is symmetric.

By using the divergence theorem and exploiting the fact that Eq. (3) must hold for all virtual velocities, we obtain

$$
\begin{gathered}
\operatorname{div} \mathbf{T}_{s}-\mathbf{m}_{s}+\mathbf{b}_{s}=\mathbf{0}, \quad \text { in } \Omega \\
-\nabla p_{f}-\mathbf{m}_{f}+\mathbf{b}_{f}=\mathbf{0}, \quad \text { in } \Omega \\
\mathbf{m}_{s}+\mathbf{m}_{f}=\mathbf{0}, \quad \text { in } \Omega \\
\mathbf{T}_{s} \mathbf{n}=\mathbf{t}_{s}, \quad \text { on } \partial \Omega \\
-p_{f} \mathbf{n}=\mathbf{t}_{f}, \quad \text { on } \partial \Omega
\end{gathered}
$$

Eqs. (4) and (5) are the momentum or force balance equations for the solid and fluid constituents, Eq. (6) expresses Newton's third law, and, Eqs. (7) and (8) are the boundary conditions of traction of the two constituents. The principle of virtual power can also allow us to take into account boundary conditions on the displace- 
(a)

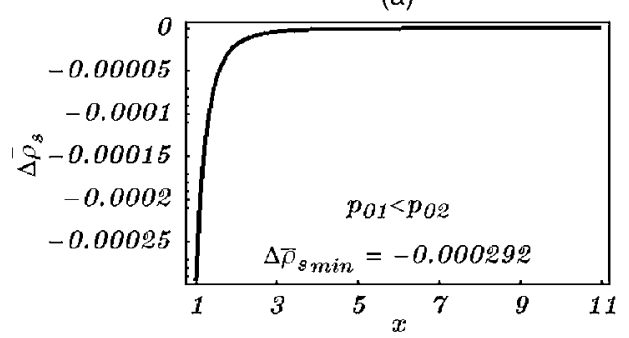

(b)

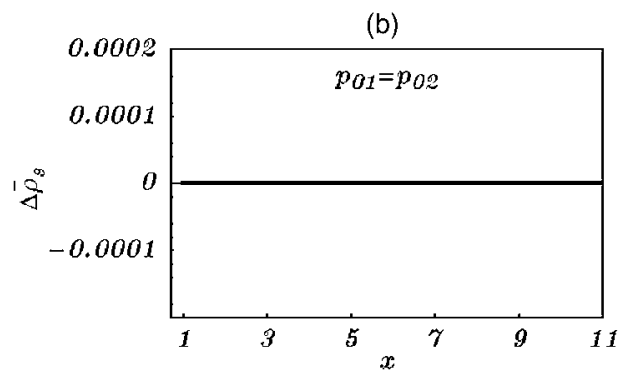

(c)

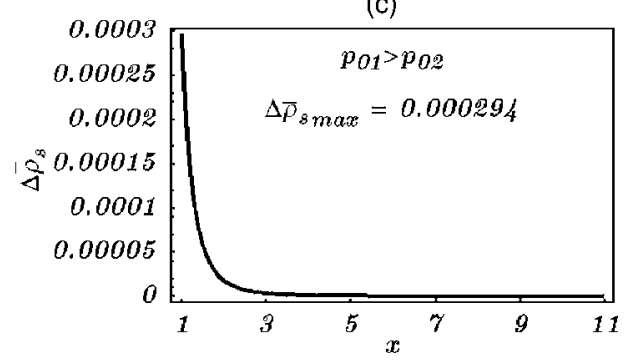

Fig. 2. Variation of the solid normalized apparent density change $\Delta \bar{\rho}_{s}=\Delta \rho_{s} / \rho_{s}^{0}$ plotted against $x$ to a change in cavern pressure $\Delta p_{01}=1 \mathrm{MPa}$. Computations were done for phenomenological constants of Table 1: (a) $p_{02}=18 \mathrm{MPa}$ and $p_{01}=7.2 \mathrm{MPa}$; (b) $p_{01}=p_{02}$; (c) $p_{01}=28.8 \mathrm{MPa}$.

ment field simply by restricting the space of virtual velocities to those fields that vanish on the boundary itself.

\section{Constitutive Relations}

The balance laws, Eqs. (4) and (5), are to be supplemented by constitutive relations; we express these in terms of the internal energy. We presume that the mixture is at a uniform temperature, the constituents are deforming quasi-statically so that their kinetic energy can be neglected, and no energy is dissipated. The internal energy density is assumed to exhibit only elastic properties of the solid and fluid constituents $\epsilon=\epsilon\left(\mathbf{F}_{s}, \rho_{f}, \mathbf{X}_{s}\right)$. Thus, the balance of energy takes the form

$$
\begin{aligned}
\frac{d_{\mathbf{v}}}{d t} \int_{\Omega} \rho \epsilon\left(\mathbf{F}_{s}, \rho_{f}, \mathbf{X}_{s}\right) d V= & \int_{\Omega}\left(\mathbf{b}_{s} \cdot \mathbf{v}_{s}+\mathbf{b}_{f} \cdot \mathbf{v}_{f}\right) d V \\
& +\int_{\partial \Omega}\left(\mathbf{t}_{s} \cdot \mathbf{v}_{s}+\mathbf{t}_{f} \cdot \mathbf{v}_{f}\right) d A
\end{aligned}
$$

in which $\mathbf{F}_{s}=$ deformation gradient of the solid; and $d_{\mathbf{v}} / d t=$ material time derivative following the barycentric motion of the mixture. According to the Reynolds transport theorem, the following constitutive equations must hold:

$$
\begin{gathered}
\mathbf{T}_{s}=\rho \frac{\partial \epsilon}{\partial \mathbf{F}_{s}} \mathbf{F}_{s}^{T} \\
p_{f}=\rho \rho_{f} \frac{\partial \epsilon}{\partial \rho_{f}} \\
\mathbf{m}_{s}=-\rho\left[\xi_{f}\left(\nabla \mathbf{F}_{s}\right)^{T} \frac{\partial \epsilon}{\partial \mathbf{F}_{s}}+\xi_{f} \mathbf{F}_{s}^{-T} \frac{\partial \epsilon}{\partial \mathbf{X}_{s}}-\xi_{s} \frac{\partial \epsilon}{\partial \rho_{f}} \nabla \rho_{f}\right]
\end{gathered}
$$

in which

$$
\xi_{f}=\frac{\rho_{f}}{\rho}, \quad \xi_{s}=\frac{\rho_{s}}{\rho}, \quad \rho=\rho_{f}+\rho_{s}
$$

are the fluid and solid mass fractions and, by definition, $\xi_{s}+\xi_{f}=1$.

In this paper we limit attention to external actions for which $\mathbf{b}_{s}=\mathbf{b}_{f}=\mathbf{0}$, i.e., only external surface tractions are applied. When applying the interior pressure of the interface between the single fluid in the cavern, a suitable procedure must be known for how to divide this traction between the two constituents. Our earlier studies (Sciarra et al. 2001) have shown that, to avoid violation of the second law of thermodynamics, the existence of a potential function must be assumed such that the working $\dot{W}^{\text {ext }}$ of the external surface tractions is given by

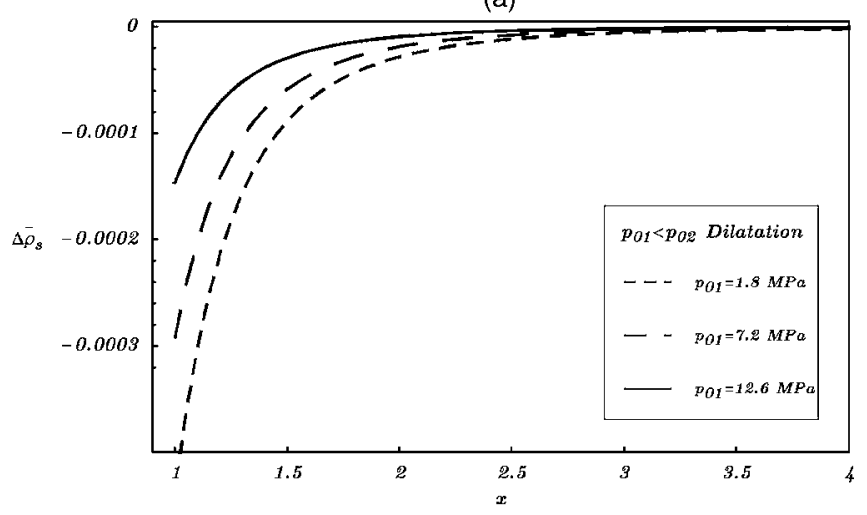

(b)

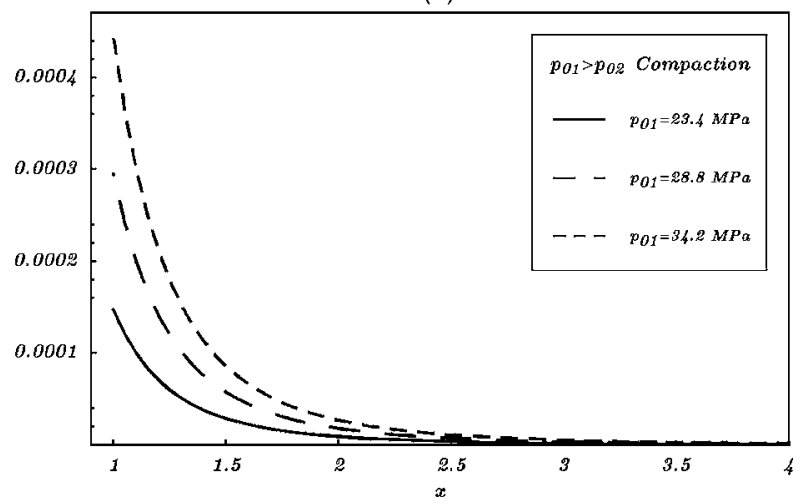

Fig. 3. Variation of the normalized solid perturbation density $\Delta \bar{\rho}_{s}=\Delta \rho_{s} / \rho_{s}^{0}$ for $\Delta p_{01}=1 \mathrm{MPa}$ and different values of the cavern pressure $p_{01}$, as indicated, and for (a) $p_{01}<p_{02}$ and (b) $p_{01}>p_{02}$. For increasing $p_{01}$, the value of $\Delta \bar{\rho}_{s}$ at the cavern wall increases and the boundary layer thickens. Computations were done with the values of Table 1 and $p_{02}=18 \mathrm{MPa}$. 


$$
\dot{W}^{\mathrm{ext}}=\frac{d_{\mathbf{v}}}{d t} \int_{\Omega} \psi^{\mathrm{ext}}\left(\mathbf{x}, \rho_{s}, \rho_{f}\right) d V
$$

where $\dot{W}^{\text {ext }}=$ surface integral on the right-hand side of Eq. (9). The external surface tractions for which Eq. (14) holds are conservative.

Requiring that Eq. (14) holds for all choices of the velocity field, we obtain

$$
\begin{aligned}
& \frac{\partial \psi^{\mathrm{ext}}}{\partial \rho_{s}}=C_{s}, \quad \frac{\partial \psi^{\mathrm{ext}}}{\partial \rho_{f}}=C_{f}, \quad \text { in } \Omega \\
& \mathbf{t}_{s}=-\frac{\partial \psi^{\mathrm{ext}}}{\partial \rho_{s}} \rho_{s}+\xi_{s} \psi^{\mathrm{ext}}, \quad \text { on } \partial \Omega \\
& \mathbf{t}_{f}=-\frac{\partial \psi^{\mathrm{ext}}}{\partial \rho_{f}} \rho_{f}+\xi_{f} \psi^{\mathrm{ext}}, \quad \text { on } \partial \Omega
\end{aligned}
$$

Eq. (15) with $C_{s}$ and $C_{f}$ as constants are necessary conditions for the existence of a $\psi^{\text {ext }}$ for which $\mathbf{b}_{s}=\mathbf{b}_{f}=\mathbf{0}$.

The preceding relations must be complemented by explicit expressions for the internal energy $\epsilon$ and the external potential $\psi^{\text {ext }}$, and both must account for the prestress acting in the reference configuration. Denoting these stresses by $\mathbf{T}_{s}^{0}$ and $p_{f}^{0}$, respectively, they must satisfy the equilibrium conditions

$$
\operatorname{div} \mathbf{T}_{s}^{0}=\mathbf{0}, \quad \nabla p_{f}^{0}=0
$$

We assume an internal energy $\epsilon$ that depends on $\mathbf{T}_{s}^{0}, \mathbf{H}_{s}=\nabla \mathbf{u}_{s}$, where $\mathbf{u}_{s}=$ solid displacement field; $\gamma_{f}$ and $\Delta \rho_{f}=\rho_{f}-\rho_{f}^{0}$, quadratic in $\mathbf{H}_{s}$ and $\Delta \rho_{f}$. This argument yields

$$
\begin{aligned}
\epsilon= & \frac{1}{\rho_{0}}\left[\mathbf{T}_{s}^{0} \cdot \mathbf{H}_{s}+\gamma_{f} \Delta \rho_{f}+\frac{1}{2} \mathbf{C}\left[\mathbf{H}_{s}\right] \cdot \mathbf{H}_{s}-\frac{1}{4}\left(\mathbf{T}_{s}^{0} \mathbf{H}_{s}^{T}-\mathbf{H}_{s} \mathbf{T}_{s}^{0}\right) \cdot \mathbf{H}_{s}\right. \\
& +\frac{1}{8}\left(\mathbf{H}_{s} \mathbf{T}_{s}^{0}-\mathbf{T}_{s}^{0} \mathbf{H}_{s}+\mathbf{T}_{s}^{0} \mathbf{H}_{s}^{T}-\mathbf{H}_{s}^{T} \mathbf{T}_{s}^{0}\right) \cdot \mathbf{H}_{s}+\frac{1}{2} \gamma_{f f} \Delta \rho_{f}^{2} \\
& \left.+K_{s f} \Delta \rho_{f} \mathbf{I} \cdot \mathbf{H}_{s}\right]
\end{aligned}
$$

in which $\rho_{0}=\left(\rho_{s}+\rho_{f}\right)_{0}=$ mixture density and $\mathbf{C}=$ elasticity tensor:

$$
\mathbf{C}\left[\mathbf{H}_{s}\right]=2 \mu \operatorname{sym}_{s}+\lambda\left(\operatorname{tr} \mathbf{H}_{s}\right) \mathbf{I}
$$

where $\lambda$ and $\mu=$ Lamé constants. Moreover, $\gamma_{f}, \gamma_{f f}$, and $K_{s f}=$ material parameters. The spherical tensor $K_{s f} \mathbf{I}$ accounts for the interaction between the solid and the fluid phases because of the deformation of the pores, while $\gamma_{f}$ and $\gamma_{f f}$ mimic fluid apparent compressibilities due to the initial pressure $p_{f}^{0}$ and the additional load $\Delta p_{f}$. A constant term has been omitted in Eq. (19). The first two members of the right-hand side of Eq. (19) are linear in $\mathbf{H}_{s}$ and $\Delta \rho_{f}$ and thus account for the primary effect of the nontrivial stress state in the reference configuration. All other terms are second order and yield a linear contribution of $\mathbf{H}_{s}$ and $\Delta \rho_{f}$ to the solid and the fluid stress. They also contain prestress contributions that may contribute to an anisotropic response behavior also if $\mathbf{C}[\cdot]$ is isotropic, as suggested by Eq. (20).

The presence of a prestress exerted in the solid as well as in the fluid phase accounts for the initial state of stress due to the lithostatic confining traction and the internal pressure of the cavern. This contribution, which is typically omitted, will allow the determination of a prediction for the disturbance induced by a change of the cavern pressure in terms of changes in the solid and fluid densities close to the cavern walls with respect to their initial values. We underline that, in the present paper, we do not explicitly discuss the range of values of $\mathbf{T}_{s}^{0}, \gamma_{f}$, and $K_{s f}$ which guarantees the second-order polynomial in Eq. (19) to be positive definite; in other words, no stability analysis has been developed. Apparently, further steps should be devoted to investigate the buckling limit of the mixture.

It has to be noticed that Eq. (19) may be obtained by means of a Taylor expansion of the free-energy truncated at the second order. Its physical interpretation must be based on the remark that, in the reference configuration, the mixture elementary volume element is not stress-free, and moreover its state of stress is not spherical. In particular, as it is very well known in the literature, the deformation energy depends on the whole deformation gradient and not only on its symmetric part.

The constitutive choice for $\psi^{\text {ext }}$ will depend upon the form of the traction applied at the cylindrical boundary and at infinity. Thus, the subsequent analysis will be specialized for the assumed state of stress that we are analyzing in this paper. It will be supposed that the deformations of the mixture are axisymmetric; i.e., in cylindrical coordinates the two in-plane components of the displacement $u_{r}$ and $u_{\theta}$ are functions of the radial coordinate $r$ only. This implies that Eq. (18) possesses the solutions

$$
\begin{gathered}
\mathbf{T}_{s}^{0}=\left(a_{0}-\frac{b_{0}}{r^{2}}\right) \mathbf{e}_{r} \otimes \mathbf{e}_{r}+\left(a_{0}+\frac{b_{0}}{r^{2}}\right) \mathbf{e}_{\theta} \otimes \mathbf{e}_{\theta} \\
p_{f}^{0}=\gamma_{f} \rho_{f}^{0}=\mathrm{const}
\end{gathered}
$$

in which $\mathbf{e}_{r}$ and $\mathbf{e}_{\theta}=$ radial and azimuthal unit vectors. Therefore, the solid stress $\mathbf{T}_{s}^{0}$ only has a radial and an azimuthal component. Its isotropic part $a_{0}$ defines a homogeneous pressure, while its deviatoric part exhibits the normal stress effects. These have the largest contribution at the cylindrical wall and decay quadratically as $r^{-2}$ as one moves to infinity. The radial stress is decreased from the ambient pressure $a_{0}$ by $b_{0} r^{-2}$; the hoop stress, on the other hand, is increased by this same amount. The fluid pressure Eq. (22) is uniformly distributed in the entire exterior of the cylinder and linearly related to the initial apparent fluid density $\rho_{f}^{0}$.

The conceivable most simple representation of $\psi^{\text {ext }}$ is of the form

$$
\begin{aligned}
\psi^{\mathrm{ext}} & =\psi_{0}^{\mathrm{ext}}+\Delta \psi^{\mathrm{ext}} \\
& =\left(C_{s} \rho_{s}^{0}+C_{f} \rho_{f}^{0}+\bar{\pi}_{0}+\frac{\bar{\pi}_{1}}{r}\right)+\left(C_{s} \Delta \rho_{s}+C_{f} \Delta \rho_{f}+\Delta \pi_{0}\right)
\end{aligned}
$$

in which $C_{s}, C_{f}=$ phenomenological constants; and $\bar{\pi}_{0}, \bar{\pi}_{1}$, and $\Delta \pi_{0}$ are related to the pressure at the boundaries in the reference and in the perturbed configuration. To find these connections, Eq. (23) is substituted into Eqs. (16) and (17). A routine, but somewhat lengthy, computation yields the solid-fluid traction vectors

$$
\begin{gathered}
\mathbf{t}_{s}^{0}=\left[-\xi_{f}^{0} \xi_{s}^{0} \rho_{0}\left(C_{s}-C_{f}\right)+\xi_{s}^{0}\left(\bar{\pi}_{0}+\frac{\bar{\pi}_{1}}{r}\right)\right] \mathbf{n}, \quad \text { at } r=a, r \rightarrow \infty \\
\mathbf{t}_{f}^{0}=\left[\xi_{f}^{0} \xi_{s}^{0} \rho_{0}\left(C_{s}-C_{f}\right)+\xi_{f}^{0}\left(\bar{\pi}_{0}+\frac{\bar{\pi}_{1}}{r}\right)\right] \mathbf{n}, \quad \text { at } r=a
\end{gathered}
$$

Because $\mathbf{t}^{0}=\mathbf{t}_{s}^{0}+\mathbf{t}_{f}^{0}$, we deduce from Eq. (24) that

$$
\mathbf{t}^{0}(a)=\left(\bar{\pi}_{0}+\frac{\bar{\pi}_{1}}{a}\right) \mathbf{n}=-p_{01} \mathbf{n}, \quad \text { at } r=a
$$




$$
\lim _{r \rightarrow \infty} \mathbf{t}^{0}(r)=\left(-\xi_{f}^{0} \xi_{s}^{0} \rho_{0}\left(C_{s}-C_{f}\right)+\xi_{s}^{0} \bar{\pi}_{0}\right) \mathbf{n}=-p_{02} \mathbf{n}, \quad \text { as } r \rightarrow \infty
$$

Finally, there remains the determination of $a_{0}$ and $b_{0}$ in Eq. (21). With the aid of Eq. (24) we may write

$$
\begin{aligned}
\left(a_{0}-\frac{b_{0}}{a^{2}}\right) \mathbf{n} & =\left[-\xi_{f}^{0} \xi_{s}^{0} \rho_{0}\left(C_{s}-C_{f}\right)+\xi_{s}^{0}\left(\bar{\pi}_{0}+\frac{\bar{\pi}_{1}}{a}\right)\right] \mathbf{n}, \quad \text { at } r=a \\
a_{0} \mathbf{n} & =\left[-\xi_{f}^{0} \xi_{s}^{0} \rho_{0}\left(C_{s}-C_{f}\right)+\xi_{s}^{0} \bar{\pi}_{0}\right] \mathbf{n}, \quad \text { as } r \rightarrow \infty
\end{aligned}
$$

from which

$$
a_{0}=\left[-\xi_{f}^{0} \xi_{s}^{0} \rho_{0}\left(C_{s}-C_{f}\right)+\xi_{s}^{0} \bar{\pi}_{0}\right], \quad b_{0}=-\xi_{s}^{0} a \bar{\pi}_{1}
$$

may be deduced, once the following expressions for $\pi_{0}$ and $\pi_{1}$ are taken into account from Eq. (25):

$$
\bar{\pi}_{0}=-\frac{p_{02}}{\xi_{s}^{0}}+\xi_{f}^{0} \rho_{0}\left(C_{s}-C_{f}\right), \quad \bar{\pi}_{1}=a \frac{p_{02}}{\xi_{s}^{0}}-a p_{01}-a \xi_{f}^{0} \rho_{0}\left(C_{s}-C_{f}\right)
$$

For $C_{s}=C_{f}$, which means that the external potential simply depends on the mixture density, Eqs. (24) and (25) simplify considerably.
Eq. (25) defines the boundary conditions for the zeroth order problem setting. In other words, for a given internal pressure $p_{01}$ of the cylindrical boundary and a prescribed outer pressure $p_{02}$, the density distributions $\rho_{f}^{0}$ and $\rho_{s}^{0}$ can be computed in terms of $p_{01}$ and $p_{02}$, once suitable constitutive relations have been taken into account for solid and fluid reference stresses. In the subsequent sections, this solution is regarded as a basic reference state from which deviations will now be determined.

\section{Perturbed Linear Boundary Value Problem}

Consider now the cylindrical cavity embedded in the solid-fluid medium subjected to the boundary pressures $p_{01}$ and $p_{02}$ as described previously. Assume that, by the application of some external action, the pressure $p_{01}$ on the boundary of the cavity is quasi-statically changed to $p_{01}+\Delta p_{01}$, while on the external boundary the pressure $p_{02}$ is kept. This can for instance be achieved by pressing liquid into the cavern or by removing it. This will change the state of stress in the surrounding solid-fluid medium. For $\Delta p_{01}>0$, the fluid will be pressed into the pore space, the pores will tend to open, and the solid component will experience a density change and a radial displacement field $u_{r}^{s}$ that will attenuate to zero as $r$ tends to infinity.

It will now be assumed that all fields depend only on the radial coordinate and that the solid displacement field $\left(u_{r}^{s}, u_{\theta}^{s}\right)$ as well as the change in densities $\Delta \rho_{f}$ and $\Delta \rho_{s}$ and pressure $\Delta \pi_{0}$ are small, such that nonlinear terms can be dropped. The linearized field equations, derivable from Eqs. (4), (5), (10), (11), and (12), then take the forms

$$
\begin{gathered}
{\left[2 \xi_{f}^{0}\left(a_{0}-\frac{b_{0}}{r^{2}}\right)+\lambda+2 \mu\right] u_{r}^{\prime \prime}+\frac{1}{r}\left[2 \xi_{f}^{0}\left(a_{0}+\frac{b_{0}}{r^{2}}\right)+\frac{2 b_{0}}{r^{2}}+\lambda+2 \mu\right] u_{r}^{\prime}+\frac{1}{r^{2}}\left[-2 \xi_{f}^{0}\left(a_{0}+\frac{b_{0}}{r^{2}}\right)-\frac{2 b_{0}}{r^{2}}-(\lambda+2 \mu)\right] u_{r}} \\
+\left[\frac{1}{\rho_{0}}\left(a_{0}-\frac{b_{0}}{r^{2}}\right)+K_{s f}-\xi_{s}^{0} \gamma_{f}\right] \Delta \rho_{f}^{\prime}=0 \\
\frac{1}{r^{2}}\left[\left(\frac{a_{0}}{2}+\mu\right) r^{2}-b_{0}\right] u_{\theta}^{\prime \prime}+\frac{1}{r^{3}}\left[\left(\frac{a_{0}}{2}+\mu\right) r^{2}-b_{0}\right] u_{\theta}^{\prime}-\frac{1}{r^{4}}\left[\left(\frac{a_{0}}{2}+\mu\right) r^{2}-b_{0}\right] u_{\theta}=0 \\
\Delta \rho_{f}^{\prime}=\frac{\rho_{f}^{0}\left[\rho_{s}^{0} \gamma_{f}-\rho_{0} K_{s f}\right]\left(u_{r}^{\prime}+\frac{1}{r} u_{r}\right)^{\prime}-\rho_{f}^{0}\left[\left(a_{0}-\frac{b_{0}}{r^{2}}\right) u_{r}^{\prime}\right]^{\prime}-\left[\rho_{f}^{0}\left(a_{0}+\frac{b_{0}}{r^{2}}\right) \frac{1}{r} u_{r}\right]^{\prime}}{2 \rho_{f}^{0} \gamma_{f}+\rho_{0} \rho_{f}^{0} \gamma_{f f}}
\end{gathered}
$$

valid in $a<r<\infty$, and the first-order boundary conditions follow from the ansatz Eq. (23) and the localizations Eqs. (16) and (17):

$$
\begin{aligned}
\Delta \mathbf{t}_{s}= & \left\{\left[-\left(\xi_{f}^{0}\right)^{2}\left(C_{s}-C_{f}\right)+\frac{\xi_{f}^{0}}{\rho_{0}}\left(\bar{\pi}_{0}+\frac{\bar{\pi}_{1}}{r}\right)\right] \Delta \rho_{s}+\left[-\left(\xi_{s}^{0}\right)^{2}\left(C_{s}-C_{f}\right)\right.\right. \\
& \left.\left.+\frac{\xi_{s}^{0}}{\rho_{0}}\left(\bar{\pi}_{0}+\frac{\bar{\pi}_{1}}{r}\right)\right] \Delta \rho_{f}+\xi_{s}^{0} \Delta \pi_{0}\right\} \mathbf{n}, \quad \text { at } r=a
\end{aligned}
$$

$$
\begin{aligned}
\Delta \mathbf{t}_{f}= & \left\{\left[\left(\xi_{s}^{0}\right)^{2}\left(C_{s}-C_{f}\right)+\frac{\xi_{s}^{0}}{\rho_{0}}\left(\bar{\pi}_{0}+\frac{\bar{\pi}_{1}}{r}\right)\right] \Delta \rho_{f}+\left[\left(\xi_{f}^{0}\right)^{2}\left(C_{s}-C_{f}\right)\right.\right. \\
& \left.\left.-\frac{\xi_{f}^{0}}{\rho_{0}}\left(\bar{\pi}_{0}+\frac{\bar{\pi}_{1}}{r}\right)\right] \Delta \rho_{s}+\xi_{f}^{0} \Delta \pi_{0}\right\} \mathbf{n}, \quad \text { at } r=a
\end{aligned}
$$




$$
\Delta p_{01}=-\Delta \pi_{0}, \quad \text { at } r=a
$$

thus identifying the perturbation $\Delta \pi_{0}$ with the perturbation pressure $\Delta p_{01}$ applied at the cylindrical wall and defining the solid and fluid boundary pressures according to

$$
\begin{aligned}
\Delta p_{s}(a)= & -\left\{\left[-\left(\xi_{f}^{0}\right)^{2}\left(C_{s}-C_{f}\right)+\frac{\xi_{f}^{0}}{\rho_{0}}\left(\bar{\pi}_{0}+\frac{\bar{\pi}_{1}}{r}\right)\right] \Delta \rho_{s}\right. \\
+ & {\left.\left[-\left(\xi_{s}^{0}\right)^{2}\left(C_{s}-C_{f}\right)+\frac{\xi_{s}^{0}}{\rho_{0}}\left(\bar{\pi}_{0}+\frac{\bar{\pi}_{1}}{r}\right)\right] \Delta \rho_{f}+\xi_{s}^{0} \Delta \pi_{0}\right\} } \\
\Delta p_{f}(a)= & -\left\{\left[\left(\xi_{s}^{0}\right)^{2}\left(C_{s}-C_{f}\right)+\frac{\xi_{s}^{0}}{\rho_{0}}\left(\bar{\pi}_{0}+\frac{\bar{\pi}_{1}}{r}\right)\right] \Delta \rho_{f}\right. \\
& \left.+\left[\left(\xi_{f}^{0}\right)^{2}\left(C_{s}-C_{f}\right)-\frac{\xi_{f}^{0}}{\rho_{0}}\left(\bar{\pi}_{0}+\frac{\bar{\pi}_{1}}{r}\right)\right] \Delta \rho_{s}+\xi_{f}^{0} \Delta \pi_{0}\right\}
\end{aligned}
$$

In addition, the vanishing shear stresses in $r=a$ require

$$
u_{\theta}^{\prime}(a)=0
$$

and the boundary conditions at $r \rightarrow \infty$ require

$$
\lim _{r \rightarrow \infty} u_{r}(r)=\lim _{r \rightarrow \infty} u_{\theta}(r)=0
$$

A detailed derivation of Eqs. (29)-(33) is given in the Appendix.

Thus, the proposed steady-state deformation problem appears mathematically as a standard linear two-point boundary value problem comprising the ordinary differential Eqs. (29)-(31) and the boundary conditions Eqs. (32), (33), (37), and (38). The differential equations are homogeneous and so are the boundary conditions Eqs. (37) and (38), but the traction boundary conditions are inhomogeneous via the prescription of $\Delta p_{01}$.

Inspection of Eqs. (30) and (37), and the second part of Eq. (38) shows that the solution for $u_{\theta}$ is decoupled from those of Eqs. (29), (31)-(33), and the first part of Eq. (38). Now, because the boundary value problem for $u_{\theta}$ is homogeneous, $u_{\theta}=0$ is a solution; in fact, this trivial result follows simply by the symmetry of the boundary value problem. What remains are the differential Eqs. (29) and (31) subjected to the boundary conditions Eqs. (32) and (33), and the first part of Eq. (38). The construction of their solution will be our next task.

\section{Solution of Boundary Value Problem}

The coupled system Eqs. (29) and (31) of ordinary differential equations can be reduced to a simple second-order differential equation for $u_{r}$ if Eq. (31) is substituted into Eq. (29). This new differential equations has the form

$$
a(r) u_{r}^{\prime \prime}+b(r) u_{r}^{\prime}+c(r) u_{r}=0
$$

with coefficients that are singular at $r=0$ and are such that Eq. (39) is not of the Fuchs type. It follows that no convergent series solutions can be found. Thus, it is hopeless to search for analytical solutions. Solutions must be constructed by numerical techniques, and these are not likely to offer any difficulties, because the only singularities of $a(r), b(r)$, and $c(r)$ lie outside the range of integration, $(a, \infty)$, namely, at $r=0$.
For the numerical integration of the differential Eqs. (29) and (31), it is advantageous to reduce it to first-order standard form and simultaneously to nondimensionalize the equations. To this end, we select

$$
r=a x, \quad u_{r}=a y_{1}, \quad \frac{d u_{r}}{d r}=y_{2}, \quad \Delta \rho_{f}=\rho_{f}^{0} y_{3}
$$

With this choice, it is straightforward to show that Eqs. (29) and (31) reduce to

$$
\mathbf{A}(x) \mathbf{Y}^{\prime}=\mathbf{B}(x) \mathbf{Y}
$$

(29) where $Y^{T}=\left(y_{1}, y_{2}, y_{3}\right)$; and

$$
\mathbf{A}(x)=\left(\begin{array}{lll}
1 & 0 & 0 \\
0 & \mathcal{A}(x) \\
0 &
\end{array}\right) \quad \mathbf{B}(x)=\left(\begin{array}{lll}
0 & 1 & 0 \\
\mathcal{B}(x) & 0 \\
& & 0
\end{array}\right)
$$

in which $\mathcal{A}(x)$ and $\mathcal{B}(x)$ can easily be given in terms of the coefficients of Eqs. (29) and (31). Inverting $\mathbf{A}(x)$, Eq. (41) may be written as

$$
\mathbf{Y}^{\prime}=\mathbf{A}(x)^{-1} \mathbf{B}(x) \mathbf{Y}=\mathbf{C}(x) \mathbf{Y}=\left(\begin{array}{lll}
0 & 1 & 0 \\
& \mathcal{C}(x) & 0 \\
& & 0
\end{array}\right)
$$

This is now the standard form of a vector-valued first-order ordinary differential equation, which must be integrated between $x$ $=1$ and $x \rightarrow \infty$. The boundary conditions to which it is subjected can be deduced from Eqs. (32) and (33), and the first part of Eq. (38), and they imply that

$$
\begin{gathered}
a_{1} y_{1}(1)+a_{2} y_{2}(1)=\mathcal{P}, \quad y_{3}(1)=\bar{y}_{3}, \quad \text { at } r=a \\
\lim _{x \rightarrow \infty} y_{1}(x)=0, \quad \text { at } r \rightarrow \infty
\end{gathered}
$$

where $\mathcal{P}$ and $\bar{y}_{3}$, as well as $a_{1}$ and $a_{2}$, are prescribed constants that can be expressed in terms of $p_{01}, p_{02}, \Delta p_{01}$, and all the constitutive parameters we have introduced.

The approach to solve Eqs. (43) and (44) was a fourth-order Runge-Kutta method combined with the shooting method. To this end the initial value problem

$$
\mathbf{Y}^{\prime}=\mathbf{C}(x) \mathbf{Y} \quad \mathbf{Y}(1)=\overline{\mathbf{Y}}
$$

was solved with arbitrarily selected $y_{1}(1)$, and this value was systematically altered until $\lim _{x \rightarrow \infty} y_{1}(x)=0$ was obtained with a preselected error $|\varepsilon| \ll 1 \ln$. The actual computation $x \rightarrow \infty$ was replaced by a large finite number $x_{\infty}=1,000$ and $\left|y_{1}\left(x_{\infty}\right)\right|<\varepsilon$ $=10^{-15}$ was chosen. Furthermore, the shooting method in a linear problem that does not exhibit stiff properties only requires three integrations to achieve the final solution. The integrations were conducted with the use of the software MATHEMATICA.

\section{Results}

Numerical solutions of the two-point boundary value problem Eqs. (43) and (44) were sought for fixed assigned values of the outside reference pressure $p_{02}$ and several values of $p_{01}$ starting at $p_{01}<p_{02}$ and ending at $p_{01}>p_{02}$; a numerical value for $p_{02}$ is the overburden pressure of a salt cavern underground, and $p_{01}$ can vary from atmospheric pressure to approximately $2 p_{02}$. Numerical values for all the phenomenological coefficients are collected in Table 1. Inspection of all the formulas in the previous section 
Table 1. Values of Parameters Used in Computation of Results: $E$ and $\nu=$ Young's Modulus and Poisson's Ratio of Solid Matrix; $\hat{\rho}_{s}^{0}$ and $\hat{\rho}_{f}^{0}=$ Densities of Solid and Fluid Constituent in Reference Configuration; $v_{s}^{0}$ and $v_{f}^{0}=$ Volume Fractions. In the Reference Configuration, the Mixture is Saturated

\begin{tabular}{lc}
\hline Constitutive parameters & $\begin{array}{c}\text { Geometrical and referential } \\
\text { state properties }\end{array}$ \\
\hline$E=200 \mathrm{MPa}$ & $\hat{\rho}_{s}^{0}=1,850 \mathrm{Kg} / \mathrm{m}^{3}$ \\
$\nu=0.33$ & $\hat{\rho}_{f}^{0}=1,300 \mathrm{Kg} / \mathrm{m}^{3}$ \\
$\gamma_{f f}=1.64 \quad 10^{6} \mathrm{Nm}^{4} / \mathrm{Kg}^{2}$ & $\nu_{s}^{0}=0.97$ \\
$C_{s}=C_{f}$ & $\nu_{f}^{0}=0.03$ \\
& $\rho_{s}^{0}=\hat{\rho}_{s}^{0} v_{s}^{0}=1,794.5 \mathrm{Kg} / \mathrm{m}^{3}$ \\
& $\rho_{f}^{0}=\hat{\rho}_{f}^{0} v_{f}^{0}=39 \mathrm{Kg} / \mathrm{m}^{3}$ \\
\hline
\end{tabular}

reveals that the constants $C_{s}$ and $C_{f}$ of the external potential only arise as $\left(C_{s}-C_{f}\right)$, so results are insensitive to the numerical values of these, if we choose $C_{s}=C_{f}$. The ensuing discussion will be restricted to this case.

Fig. 2 displays the dimensionless solid density change $\Delta \rho_{s} / \rho_{s}^{0}$ plotted against the distance $x=r / a$ for the particular values $p_{02}=18 \mathrm{MPa}$ and $p_{01}=7.2 \mathrm{MPa}<p_{02} \quad[$ Fig. 2(a)], $p_{01}=28.8 \mathrm{MPa}>p_{02}$ [Fig. 2(c)]. Fig. 2(b) shows the results for $p_{01}=p_{02}$, and it states that $\Delta \rho_{s}=0$ for all $x \in[1, \infty)$, which is a trivial result. The two load cases $p_{01}<p_{02}$ and $p_{01}>p_{02}$ demonstrate qualitatively different physical behavior. Indeed, for the first case, Fig. 2(a), the numerical values show $\Delta \rho_{s}<0$. This means that the pore space becomes bigger, $\Delta v_{f}>0$, where $v_{f}$ is the porosity, corresponding to a dilatation. For the second case, $\Delta \rho_{s}>0$ for all $x \in[1, \infty)$, implying that the pore space becomes smaller, $\Delta v_{f}<0$, corresponding to a compaction. As a function of $x$, this behavior is monotonous; i.e., the dilatation and compaction, respectively, decrease with increasing $x$ and seem to approach zero as $x \rightarrow \infty$.

When varying $p_{01}$ parametrically from $p_{01}=1.8 \mathrm{MPa}<p_{02}$ in steps $\delta p_{01}=5.4 \mathrm{MPa}$ until $p_{01}=34.2 \mathrm{MPa}>p_{02}$, the graphs analogous to those of Fig. 2 are as shown in Fig. 3. Evidently, the maximum value of the dilatation/compaction occurs at the cylinder wall, and it increases until increasing the difference $\left|p_{01}-p_{02}\right|$. Moreover, as $\left|p_{01}-p_{02}\right|$ increases, the boundary layer with appreciable values of the dilatation/compaction thickens. Thus, the larger the difference $\left|p_{01}-p_{02}\right|$ are, the less localized the reactions to this difference will be. This behavior is evidently consistent with the identically vanishing solution of the apparent density, which is reached at $p_{01}=p_{02}$. An estimate of the boundary layer thickness (the thickness $\delta$ of the boundary layer is the distance from the cylinder wall at which the derivative of $\Delta \rho_{s}$ becomes vanishing) has been determined computationally and is summarized in Fig. 4.

The preceding results may equally be explained somewhat differently. To this end, consider a representative elementary volume (REV) comprising idealistically of four solid quadrants and a fluid sphere in the middle with connections to the REV sides (Fig. 5). When $p_{01}<p_{02}$ [Fig. 5(a)], the dilatation is associated with an increase of the fluid volume that is filled by fluid injection from the neighboring elements or the cavern fluid. However, the true solid density and therefore the solid volume in the REV remain the same. The pressure of the solid-essentially the negative trace of the solid stress-is positive, a compression. Thus, the change of the apparent density of the solid is negative $\Delta \rho_{s}<0$. The effect of the fluid via its seepage flow is to enlarge the porosity, $\Delta v_{f}>0$, because fluid is injected.

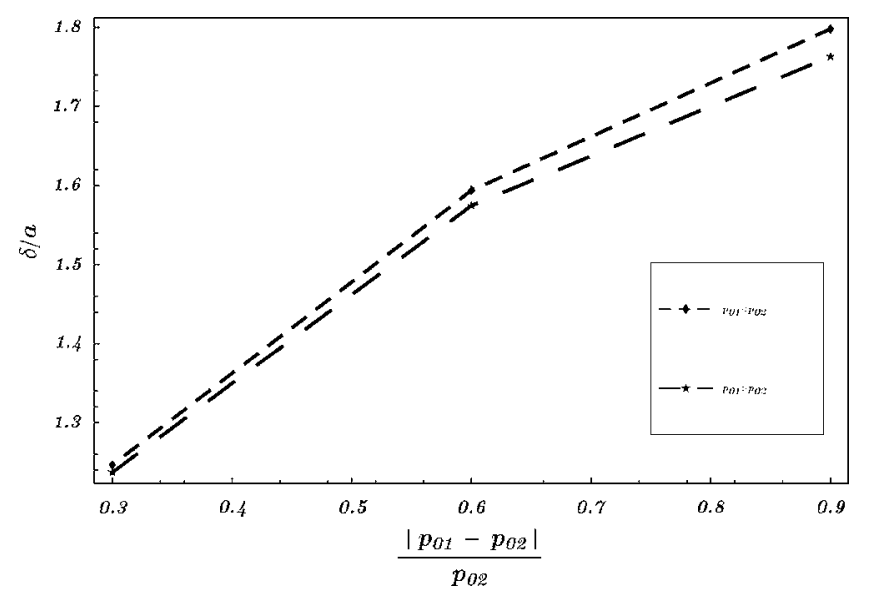

Fig. 4. Scaled boundary layer thickness $\delta / a$ plotted against the scaled initial stress difference for $p_{02}=18 \mathrm{MPa}$ and $p_{01}$ parametrically varying from $p_{01}=1.8 \mathrm{MPa}<p_{02}$ in steps $\delta p_{01}=5.4 \mathrm{MPa}$ until $p_{01}=34.2 \mathrm{MPa}>p_{02}$. For $\left|p_{01}-p_{02}\right| \rightarrow 0$, the thickness, of the boundary layer tends to zero $\delta / a \rightarrow 0$.

When $p_{01}>p_{02}$ [ Fig. 5(b)], the compaction is achieved by a reduction of the pore space, $\Delta v_{f}<0$, in the $\mathrm{REV}$, and fluid is squeezed out. The solid stress now is under tension, but the true solid density does not change so that $\Delta \rho_{s}>0$.

In both cases this behavior is the result of seepage flow and effects of the fluid rather than deformation effects in the solid component within the REV.

Accompanied with the changes in $\Delta \rho_{s}$ are also changes in the fluid pressure, $\Delta p_{f}$. Fig. 6 shows graphs for $\Delta p_{f} / p_{01}$ against $x$ for the same conditions as Fig. 2. For $p_{01}<p_{02}$ [Fig. 6(a)], the perturbation in the fluid pressure is largest at the cylinder wall, and it decreases monotonically to a nonzero value as $x \rightarrow \infty$. Alternatively, for $p_{01}>p_{02}$ [Fig. 6(b)], the perturbation pressure takes its minimum value at the cylinder wall and approaches a large asymptotic value as $x \rightarrow \infty$. In both cases typical boundary layer effects are seen.

This behavior, too, can be envisaged by sketches of typical REVs. We start from a reference configuration of the solid-fluid mixture, of which the pore structure is characterized by a positive or a negative porosity gradient when the confining state of stress satisfies the condition $p_{01}<p_{02}[$ Fig. $7(\mathrm{~b})]$ or $p_{01}>p_{02}$ [Fig. $7(d)]$, respectively. This kind of equilibrium configuration for the fluid-saturated rock salt can be achieved, for instance, as the ultimate fate of a transient creep process tending to stabilize after a suitable length of time (Cristescu and Hunsche 1998) [see, e.g., the transition from Figs. 7(a and c) to Figs. 7(b and d)]; local creep deformations enhance the convergence of the solid within the REV and consequently the closure of the pores. The smaller the confining pressure, the more relevant this effect is; for this reason, we can appreciate a positive or negative gradient of porosity in the reference configuration when $p_{01}<p_{02}$ or $p_{01}>p_{02}$, respectively. This hypothesis, endowed with the assumption of uniform apparent densities of the solid and fluid constituent in the initial equilibrium configuration, evidently implies the true densities $\hat{\rho}_{a}^{0}=\rho_{a}^{0} / \nu_{a}^{0}(a=s, f)$ not to be uniform with $r$.

The deformation of the considered reference configuration is indeed consistent with the aforementioned results regarding the fluid pressure $\Delta p_{f}$ due to a positive $\Delta p_{01}$. As we are dealing with a linear elastic model, the positive pressure $\Delta p_{01}$, applied on the 
(a)

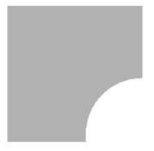

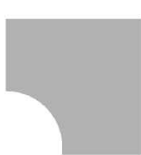

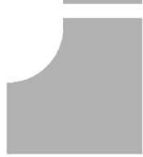

(b)
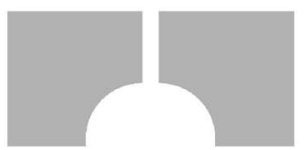

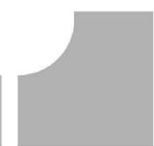

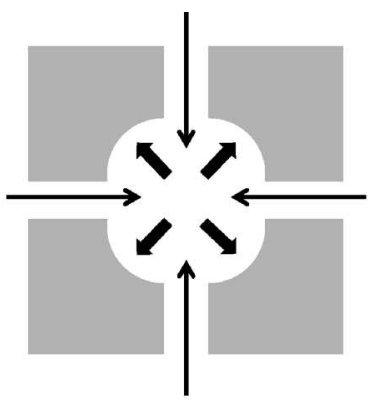

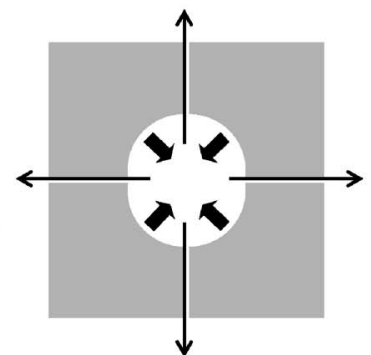

REV expands

and the solid

true density is

mantained

$\Delta \rho_{\mathrm{s}}<0, \Delta \mathrm{v}_{\mathrm{f}}>0$

$p_{01}<p_{02}$

REV contracts

and the solid

true density is

mantained

$\Delta \rho_{\mathrm{s}}>0, \Delta v_{\mathrm{f}}<0$

$p_{01}>p_{02}$

Fig. 5. Representative elementary volume (REV) with solid and fluid (interconnected with neighboring cells) in the reference configuration (left) and in the present configuration (right): (a) shows behavior when $p_{01}<p_{02}$ : the REV expands by fluid injection rather than solid deformation and thus yields an increase of the pore space; and (b) shows behavior when $p_{01}>p_{02}$ : the REV contracts and fluid is squeezed out

boundary of the cavity, is likely to imply an injection of fluid in the connected pores of the salt matrix.

Bearing in mind the previous results on the solid density profiles, let us try to discuss what happens to porosity when incompressible small deformations of the solid constituent $\left(\Delta \hat{\rho}_{s}=0\right)$ take place; apparently, the following conditions have to be fulfilled:

$$
\begin{gathered}
\Delta \rho_{s}=\Delta\left(\hat{\rho}_{s} v_{s}\right)=\Delta \hat{\rho}_{s} v_{s}^{0}+\hat{\rho}_{s}^{0} \Delta v_{s}=-\hat{\rho}_{s}^{0} \Delta v_{f} \\
\nabla \Delta \rho_{s}=\nabla \Delta\left(\hat{\rho}_{s} v_{s}\right)=-\nabla\left(\hat{\rho}_{s}^{0} \Delta v_{f}\right)=-\left(\nabla \hat{\rho}_{s}^{0}\right) \Delta v_{f}-\hat{\rho}_{s}^{0} \nabla \Delta v_{f}
\end{gathered}
$$

Because the porosity in each point $X$ of the mixture corresponds to the ratio between the fluid volume in the $\operatorname{REV}\left(V_{f}\right)$ at $X$ and the

(a)

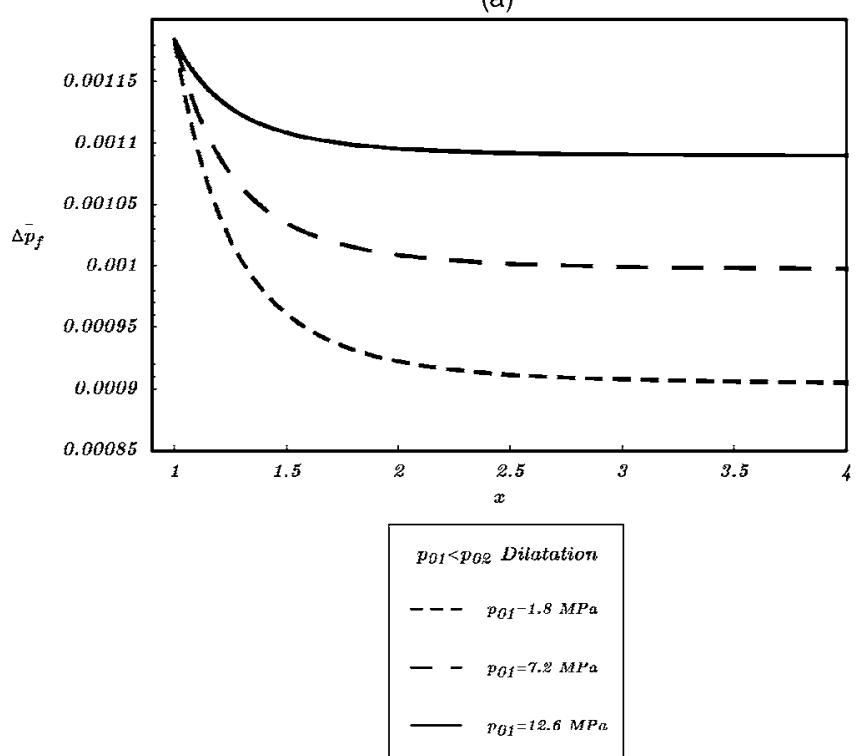

volume of the $\operatorname{REV}(V)$ itself, the following allusive chain of equalities can be established:

$$
\begin{aligned}
\Delta \nu_{f}=\Delta\left(\frac{V_{f}}{V}\right) & =\frac{\Delta V_{f}}{V_{0}}-\frac{V_{f}^{0}}{V_{0}^{2}} \Delta V=\frac{\Delta V_{f}}{V_{0}}-v_{f}^{0} \frac{\Delta V}{V_{0}} \\
\nabla\left(\Delta \nu_{f}\right) & =\nabla\left(\frac{\Delta V_{f}}{V_{0}}\right)-\nabla\left(v_{f}^{0} \frac{\Delta V}{V_{0}}\right) \\
& =\nabla\left(\frac{\Delta V_{f}}{V_{0}}\right)-v_{f}^{0} \nabla\left(\frac{\Delta V}{V_{0}}\right)-\nabla v_{f}^{0} \frac{\Delta V}{V_{0}}
\end{aligned}
$$

where $\Delta v_{f}=v_{f}-v_{f}^{0}$, i.e., the difference between the current and the

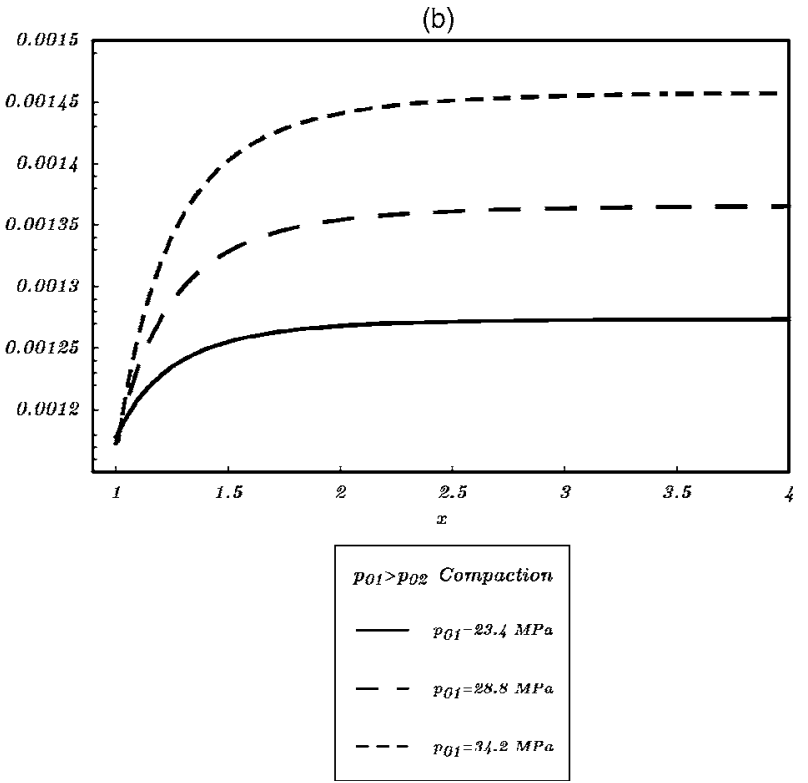

Fig. 6. Variation of the normalized fluid pressure $\Delta p_{f} / p_{02}$ (calculated for $\Delta p_{01}=1 \mathrm{MPa}$ ) plotted against $x$ for the same conditions as Fig. 3 was constructed: (a) for the dilatation case, $p_{01}<p_{02}$; and (b) for the compaction case, $p_{01}>p_{02}$. The typical boundary layer behavior is seen. 
(a)

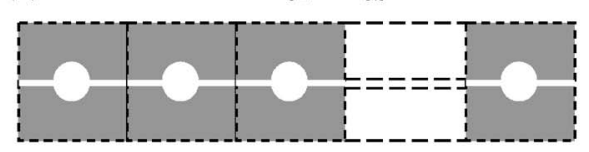

(b)

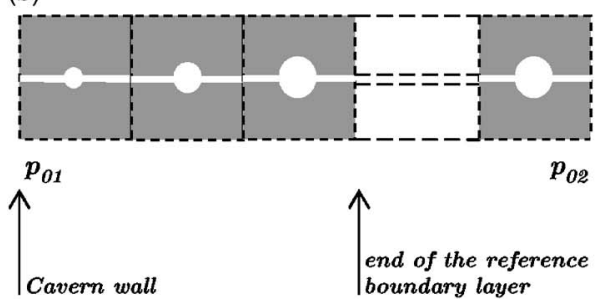

(c)

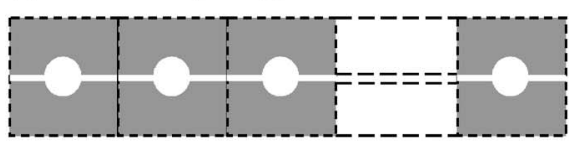

(d)

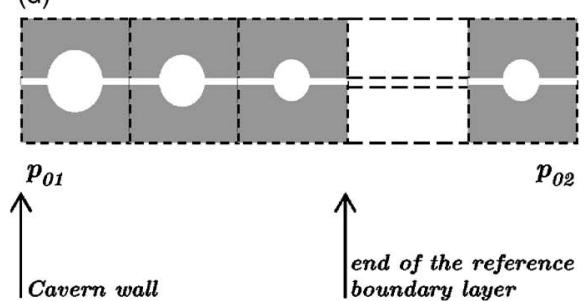

Fig. 7. Rows of representative elementary volumes from the cavern wall through to infinity. The first row shows them before any load is applied with uniform solids and fluids fraction. When $p_{01}$ and $p_{02}$ are applied from the left and right, respectively, the difference in $p_{01}$ and $p_{02}$ induces an inhomogeneous distribution of the pore space. For (b) $p_{01}<p_{02}$, the porosity increases with distance from the cavern wall; for (d) $p_{01}>p_{02}$, it decreases. This corresponds to a fluid pressure change $\Delta p_{f}$ that is decreasing [when starting from (a) the reference configuration toward (b) the current configuration panel] and increasing [when starting from (c) the reference configuration toward (d) the current configuration panel], respectively, with $x$.

reference porosity. Consider incompressible small deformations of the solid constituent $\left(\Delta \hat{\rho}_{s}=0\right)$; the variation of the fluid volume in the REV consequently provides the condition

$$
0=\Delta \hat{\rho}_{s}=-\frac{\hat{\rho}_{s}^{0}}{V_{s}^{0}} \Delta V_{s} \Rightarrow \Delta V=\Delta V_{f}
$$

which implies, because of Eqs. (48) and (49)

$$
\begin{gathered}
\Delta v_{f}=\left(1-v_{f}^{0}\right) \frac{\Delta V}{V_{0}} \\
\nabla\left(\Delta v_{f}\right)=\left(1-v_{f}^{0}\right) \nabla\left(\frac{\Delta V}{V_{0}}\right)-\nabla v_{f}^{0} \frac{\Delta V}{V_{0}}
\end{gathered}
$$

For $p_{01}<p_{02}$, the reference porosity gradient is positive $\left(\nabla \nu_{f}^{0}>0\right)$; moreover, the dilatation of the $\operatorname{REV}\left(\Delta \rho_{s}<0\right.$, say, $\left.\Delta v_{f}>0\right)$ progressively decreases $\left(\nabla \Delta \rho_{s}>0\right)$ when $r \rightarrow \infty$. Incompressible small deformations of the solid constituent imply, according to Eq. (47), the variation of porosity to decrease with $r$, say, $\nabla\left(\Delta \nu_{f}\right)<0$, as $\nabla \hat{\rho}_{s}^{0}=\rho_{s}^{0} /\left(\nu_{s}^{0}\right)^{2} \nabla \nu_{f}^{0}>0$ [see Fig. $7($ a and c)]. When the internal confining pressure is smaller than the external one, dilatancy occurs, and this effect progressively reduces going away from the cavern wall. As the behavior of the apparent density of the solid is essentially triggered by the change in volume of the REVs, the inequalities $\Delta V / V_{0}>0$ and $\nabla\left(\Delta V / V_{0}\right)<0$ must hold true, and therefore $\Delta V_{f} / V_{0}>0$ and $\nabla\left(\Delta V_{f} / V_{0}\right)<0$, because of Eq. (50). Thus, the macroscopic behavior described in Fig. 2(a) is consistent with the microinterpretation given by Eq. (52).

On the other hand, when $p_{01}>p_{02}$, the reference porosity gradient is negative $\left(\nabla v_{f}^{0}<0\right)$; moreover, compaction of the REV $\left(\Delta \rho_{s}>0\right.$, say, $\left.\Delta v_{f}<0\right)$ progressively decreases $\left(\nabla \Delta \rho_{s}<0\right)$ as $r$ tends to infinity. Incompressible small deformations of the solid constituent $\left(\Delta \hat{\rho}_{s}=0\right)$ require, according to Eq. (47), the following condition to hold true:

$$
\frac{\nabla \Delta \nu_{f}}{\left|\Delta \nu_{f}\right|}>\frac{\nabla v_{f}^{0}}{1-v_{f}^{0}}, \quad \nabla v_{f}^{0} /\left(1-v_{f}^{0}\right)<0
$$

which does not univocally determine the sign of $\nabla \Delta v_{f}$. As the behavior of the apparent density of the solid is essentially triggered by the change in volume of the REVs, the inequalities
$\Delta V / V_{0}<0\left(\Delta V_{f} / V_{0}<0\right)$ and $\nabla\left(\Delta V / V_{0}\right)>0\left(\nabla\left(\Delta V_{f} / V_{0}\right)>0\right)$ must hold true; these conditions are really consistent with the previous remarks on the sign of the gradient of porosity, according to Eq. (52).

As the mean pressure of the fluid $p_{f}$ can be essentially characterized, via a "naive" micro-macro identification, by the product $p v_{f}$, where $p$ indicates the porewater pressure, the variation of the fluid pressure $\Delta p_{f}$ will be

$$
\Delta p_{f}=p_{0} \Delta v_{f}+\Delta p v_{f}^{0}
$$

The pressure of the fluid at the micro level is essentially constant $\left(\nabla p=\nabla\left(p_{0}+\Delta p\right)=0\right)$. When $p_{01}<p_{02}$, the injection of a small amount of fluid mass into the cavern implies $\nabla \Delta p_{f}<0$ [see Fig. 6(a)], and consequently

$$
\nabla \Delta v_{f}<-\frac{\Delta p}{p_{0}} \nabla v_{f}^{0}<0
$$

which is consistent with Eq. (52); on the other hand, when $p_{01}>p_{02}$, the injection of the same amount of fluid into the cavern implies $\nabla \Delta p_{f}>0$ [see Fig. 6(b)], and consequently

$$
\nabla \Delta v_{f}>-\frac{\Delta p}{p_{0}} \nabla v_{f}^{0}>0
$$

which is consistent with Eq. (53).

\section{Conclusion}

In this paper, an analysis of the deformational behavior of the solid-fluid surrounding ground of a fluid filled cavern was pre- 
sented, when this material is subjected to a confining isotropic stress $p_{02}$ from infinity and a different pressure $p_{01}$ from the circular cavern wall. Both constituents are treated as elastic, but the linear law takes the prestress that is established by the external pressures $p_{01}$ and $p_{02}$ into account. The model is set up only to generate radial dependencies of the process variables when the cavern pressure is changed from $p_{01}$ to $p_{01}+\Delta p_{01}$, where, owing to linearity, it suffices to analyze $\Delta p_{01}=1$.

The results show that the solid density change, $\Delta \rho_{s}$, associated with $\Delta p_{01}=1$, as a function of the radial distance from the cavern is a dilatation (compaction) if $p_{01}<p_{02}\left(p_{01}>p_{02}\right)$ (Fig. 2), with the maximum modulus arising at the cavern wall and quickly tapering off, thus giving rise to a boundary layer of pore space opening (or closure) close to the cavern wall. This nontrivial dilatancy is solely due to the state of prestress, and it collapses when $p_{01}=p_{02}$ and when $\Delta \rho_{s}=0$ for $r>a$.

We note that the parametric analysis which has been developed represents a first step toward the study of the behavior of salt caverns, in particular when the internal pressure is greater than the lithostatic one. We noticed that the dilatancy phenomenon, induced by the injection of a liquid mass into the cavern, can occur, in the elastic regime, if the internal pressure is smaller than the external one. On the other hand, when the initial pressure of the cavern is greater than the external one, compaction is detected in the elastic regime. Apparently, dilatancy phenomena can also occur when reaching these pressures inside the cavern once the yield limit of the salt had been surpassed, but this is a wellknown issue. We prove that, in order to avoid dilatancy, it is not sufficient to keep the internal pressure below the lithostatic limit but also the conditions to assure an elastic regime have to be investigated.

Our analysis has obviously been restrictive in several respects and ought to be extended. First, the state of prestress does not contain shear stresses and may thus overlook a significant state of stress arising in practical applications. For instance, at the far-end boundaries, the vertical and horizontal confining pressures would differ from one another and, thus, induce a combination of an isotropic pressure plus a pure shear. Its analysis will be rather complicated. Second, the solutions of the linearly perturbed problem have not been analyzed with regard to their stability. More specifically, depending upon the initial state of stress, the perturbed solution may be linearly stable or unstable. Delineating the ranges of these stabilities (instabilities) is practically significant.

After the generalizations and improvements just delineated, it will also become possible to cope with the problem of formulating and applying a pore-plasticity model to describe more precisely the behavior of the disturbed rock zone around cavities.

\section{Acknowledgment}

This paper was written while the third writer was on sabbatical leave at the University of Rome, La Sapienza, the support of which is hereby acknowledged.

\section{Appendix.}

The constitutive Eqs. (10)-(12), together with the internal energy constitutive relation Eq. (19), are specialized bearing in mind the axisymmetric assumption of the deformation of the mixture; as in cylindrical coordinates, if the two in-plane components of the displacement $u_{r}$ and $u_{\theta}$ depend only on $r$, we have

$$
\begin{gathered}
\mathbf{T}_{s}=\frac{\Delta \rho_{f}}{\rho_{0}} \mathbf{T}_{s}^{0}-\xi_{s}^{0}\left(\operatorname{tr} \mathbf{H}_{s}\right) \mathbf{T}_{s}^{0}+\frac{1}{2}\left(\mathbf{T}_{s}^{0} \mathbf{H}_{s}^{T}+\mathbf{H}_{s} \mathbf{T}_{s}^{0 T}\right) \\
+\frac{1}{2}\left(\mathbf{W}_{s} \mathbf{T}_{s}^{0}-\mathbf{T}_{s}^{0} \mathbf{W}_{s}\right)+\mathbf{C}\left[\mathbf{H}_{s}\right]+\Delta \rho_{f} K_{s f} \mathbf{I} \\
p_{f}=\left[\gamma_{f}\left(1+\xi_{f}^{0}\right)+\rho_{f}^{0} \gamma_{f f}\right] \Delta \rho_{f}+\rho_{f}^{0}\left(K_{s f}-\xi_{s}^{0} \gamma_{f}\right) \operatorname{tr} \mathbf{H}_{s} \\
\mathbf{m}_{s}=-\xi_{f}^{0} \nabla\left(\mathbf{T}_{s}^{0} \cdot \mathbf{H}_{s}\right)+\xi_{s}^{0} \gamma_{f} \nabla\left(\Delta \rho_{f}\right)
\end{gathered}
$$

These last yield the following formulas for the divergence of the solid stress tensor and the gradient of the fluid pressure:

$$
\begin{aligned}
\operatorname{div} \mathbf{T}_{s}= & \mathbf{T}_{s}^{0}\left[\frac{1}{\rho_{0}} \nabla\left(\Delta \rho_{f}\right)-\xi_{s}^{0} \nabla\left(\operatorname{tr} \mathbf{H}_{s}\right)\right]+\frac{1}{2}\left(\nabla \mathbf{T}_{s}^{0}\right) \mathbf{H}_{s}^{T}+\frac{1}{2} \mathbf{T}_{s}^{0} \operatorname{div} \mathbf{H}_{s}^{T} \\
& +\frac{1}{2}\left(\nabla \mathbf{H}_{s}\right) \mathbf{T}_{s}^{0}+\frac{1}{2}\left(\nabla \mathbf{W}_{s}\right) \mathbf{T}_{s}^{0}+\frac{1}{2} \mathbf{T}_{s}^{0} \operatorname{div} \mathbf{W}_{s}+\operatorname{div} \mathbf{C}\left[\mathbf{H}_{s}\right] \\
& +\nabla\left(\Delta \rho_{f}\right) K_{s f} \\
\nabla p_{f} & =\left[\gamma_{f}\left(1+\xi_{f}^{0}\right)+\rho_{f}^{0} \gamma_{f f}\right] \nabla\left(\Delta \rho_{f}\right)+\rho_{f}^{0}\left(K_{s f}-\xi_{s}^{0} \gamma_{f}\right) \nabla\left(\operatorname{tr} \mathbf{H}_{s}\right)
\end{aligned}
$$

Eqs. (60) and (61), endowed with the following representation of the displacement gradient $\mathbf{H}_{s}$, assumed to depend just on $r$ :

$$
\mathbf{H}_{s}=u_{r, r} \mathbf{e}_{r} \otimes \mathbf{e}_{r}+u_{\theta, r} \mathbf{e}_{r} \otimes \mathbf{e}_{\theta}-\frac{1}{r} u_{\theta} \mathbf{e}_{\theta} \otimes \mathbf{e}_{r}+\frac{1}{r} u_{r} \mathbf{e}_{\theta} \otimes \mathbf{e}_{\theta}
$$

imply the field Eqs. (29)-(31), once the radial and the azimuthal components of Eq. (4) as well as Eq. (5) are taken into account. Note that we adopt the following rule for the tensor product $(a \otimes b) u=(a \cdot u) b$.

Boundary conditions Eqs. (32) and (33) are then achieved, starting from the external potential $\psi^{\text {ext }}[$ Eq. (23)] and its partial derivatives [Eq. (15)] with respect to the solid and fluid apparent densities, by replacing them into Eqs. (16) and (17).

\section{References}

Cosenza, P., Ghoreychi, M., Bazargan-Sabet, B., and de Marsily, G. (1999). "In situ rock salt permeability measurement for long term safety assessment of storage." Int. J. Rock Mech. Min. Sci., 36, 509-526.

Cristescu, N. D., and Hunsche, U. (1998). Time effects in rock mechanics, Wiley, Chichester, U.K.

dell'Isola, F., Guarascio, M., and Hutter, K. (2000). "A variational approach for the deformation of a saturated porous solid. A second gradient theory extending Terzaghi's effective stress principle." 
Arch. Appl. Mech., 70, 323-337.

Hutter, K., and Jöhnk, K. (2003). Continuous methods of physical modeling, Springer, Berlin.

Müller, I. (1985). Thermodynamics, Pittman, Boston.

Sciarra, G., Hutter, K., and Maugin, G. A. (2003). "A variational approach to a micro-structured theory of solid-fluid mixtures." Arch. Appl. Mech., 73, 199-224.

Sciarra, G. (2002). "Modelling of a fluid flux through a solid deformable matrix." PhD thesis, Università di Roma "La Sapienza" and Univer- sité de Toulon et du Var, Rome.

Sciarra, G., dell'Isola, F., and Hutter, K. (2001). "A solid-fluid mixture model allowing for solid dilatation under external pressure." Contr. Cybernet., 13, 287-306.

Stormont, J. C. (1997). "In situ gas permeability measurements to delineate damage in rock salt." Int. J. Rock Mech. Min. Sci., 34, 1055-1064.

Truesdell, C. (1957). "Sulle basi della termomeccanica. Nota I e II." Lincei-Rend. Sc. fis. mat. e nat. XXII (in Italian). 